\title{
A Triple AGN in a Mid-infrared Selected Late-stage Galaxy Merger
}

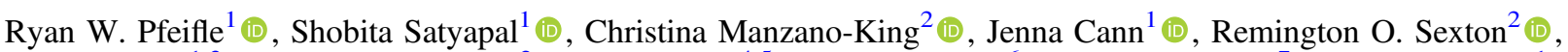

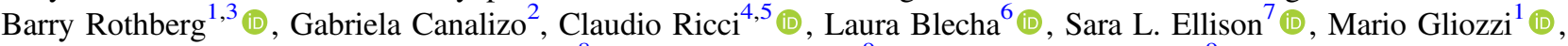 \\ Nathan J. Secrest ${ }^{8}$ (D), Anca Constantin ${ }^{9}$ (D), and Jenna B. Harvey ${ }^{9}$ \\ ${ }^{1}$ Department of Physics and Astronomy, George Mason University, MS3F3, 4400 University Drive, Fairfax, VA 22030, USA; rpfeifle@masonlive.gmu.edu \\ ${ }^{2}$ Department of Physics and Astronomy, University of California, Riverside, 900 University Avenue, Riverside, CA, 92521, USA \\ ${ }^{3}$ LBT Observatory, University of Arizona, 933 N. Cherry Avenue, Tucson, AZ 85721, USA \\ ${ }^{4}$ Núcleo de Astronomía de la Facultad de Ingeniería, Universidad Diego Portales, Av. Ejército Libertador 441, Santiago, Chile \\ ${ }^{5}$ Kavli Institute for Astronomy and Astrophysics, Peking University, Beijing 100871, People's Republic of China \\ ${ }^{6}$ Department of Physics, University of Florida, P.O. Box 118440, Gainesville, FL 32611-8440, USA \\ ${ }^{7}$ Department of Physics and Astronomy, University of Victoria, Victoria, BC V8P 1A1, Canada \\ ${ }^{8}$ U.S. Naval Observatory, 3450 Massachusetts Avenue NW, Washington, DC 20392, USA \\ ${ }^{9}$ Department of Physics and Astronomy, James Madison University, Harrisonburg, VA 22807, USA \\ Received 2019 May 24; revised 2019 July 26; accepted 2019 August 7; published 2019 October 1
}

\begin{abstract}
The coevolution of galaxies and the supermassive black holes (SMBHs) at their centers via hierarchical galaxy mergers is a key prediction of $\Lambda \mathrm{CDM}$ cosmology. As gas and dust are funneled to the SMBHs during the merger, the SMBHs light up as active galactic nuclei (AGNs). In some cases, a merger of two galaxies can encounter a third galaxy, leading to a triple merger, which would manifest as a triple AGN if all three SMBHs are simultaneously accreting. Using high spatial resolution X-ray, near-IR, and optical spectroscopic diagnostics, we report here a compelling case of an AGN triplet with mutual separations $<10 \mathrm{kpc}$ in the advanced merger SDSS J084905.51+111447.2 at $z=0.077$. The system exhibits three nuclear X-ray sources, optical spectroscopic line ratios consistent with AGN in each nucleus, a high excitation near-IR coronal line in one nucleus, and broad Pa $\alpha$ detections in two nuclei. Hard X-ray spectral fitting reveals a high column density along the line of sight, consistent with the picture of late-stage mergers hosting heavily absorbed AGNs. Our multiwavelength diagnostics support a triple AGN scenario, and we rule out alternative explanations such as star formation activity, shock-driven emission, and emission from fewer than three AGN. The dynamics of gravitationally bound triple SMBH systems can dramatically reduce binary SMBH inspiral timescales, providing a possible means to surmount the "Final Parsec Problem." AGN triplets in advanced mergers are the only observational forerunner to bound triple SMBH systems and thus offer a glimpse of the accretion activity and environments of the AGNs prior to the gravitationally bound triple phase.
\end{abstract}

Key words: galaxies: active - galaxies: evolution - infrared: galaxies - X-rays: galaxies

\section{Introduction}

Observational campaigns and theoretical studies have shown both that supermassive black holes (SMBHs) reside at the centers of most galaxies and that galaxy interactions are ubiquitous in the universe. As a result, galaxies grow and evolve hierarchically through collisions (e.g., Toomre \& Toomre 1972; Schweizer 1982, 1996; Barnes \& Hernquist 1992; Hibbard \& van Gorkom 1996; Rothberg \& Joseph 2004), during which gravitational torques drive gas reservoirs toward the centers of each galaxy, potentially fueling the SMBHs at their centers (Barnes \& Hernquist 1992; Mihos \& Hernquist 1996; Hopkins et al. 2008; Blecha et al. 2018). Recent simulations predict that late-stage galaxy mergers-with nuclear pair separations $\lesssim 10 \mathrm{kpc}$-facilitate the most rapid black hole growth and represent the merger stage during which both SMBHs are expected to begin accreting as active galactic nuclei (AGNs; Hopkins et al. 2008; Blecha et al. 2018). Such dual AGNs are predicted to be highly obscured by gas and dust, consequently exhibiting red mid-infrared colors (Blecha et al. 2018). Indeed, observational studies have demonstrated that late-stage galaxy mergers (pair separations $<10 \mathrm{kpc}$ ) and postmergers host higher fractions of dust obscured AGNs than do isolated galaxies in rigorously matched control samples, and in fact mid-infrared selection identifies a larger quantity of obscured AGNs in mergers than traditional optical selection techniques (Satyapal et al. 2014; Weston et al. 2017; Ellison et al. 2019). Despite their important role in black hole and galaxy evolution, however, dual AGNs are rare systems, and less than 30 have been robustly verified in the literature (see Table 8 in Satyapal et al. 2017 and references therein). The rarity of dual AGNs emphasizes the need for efficient selection methods when conducting systematic searches for dual AGNs.

In our previous studies of dual AGNs, we preselected the latestage galaxy mergers (pair separations $<10 \mathrm{kpc}$ ) based upon their mid-infrared Wide-field Infrared Survey Explorer (WISE) colors (Satyapal et al. 2017; Pfeifle et al. 2019), selecting systems that exhibit a WISE W1 [3.4 $\mu \mathrm{m}]-W 2[4.6 \mu \mathrm{m}]>0.5$ color, a color cut that has been demonstrated in simulations to be the most effective at identifying AGNs in late-stage mergers (Blecha et al. 2018). We obtained follow-up high spatial resolution X-ray observations of these mergers with the Chandra $X$-ray Observatory (Chandra) and longslit near-infrared spectroscopic observations with the Large Binocular Telescope (LBT) to look for dual nuclear X-ray sources and high ionization coronal emission lines. Not only did a majority of the mergers host dual AGNs or dual AGN candidates (Ellison et al. 2017; Satyapal et al. 2017; Pfeifle et al. 2019), but one system exhibited three nuclear X-ray sources and-upon closer inspection-was realized as a clear case of a triple merger: SDSS 
J084905.51+111447.2 (henceforth SDSS J0849+1114). ${ }^{10}$ This study demonstrated the effectiveness of using mid-infrared colors as a preselection strategy for finding AGNs in advanced mergers.

Dual AGNs in advanced mergers represent the most observationally accessible progenitors of the SMBH binary phase, which likely produces the main source of gravitational waves (Wyithe \& Loeb 2003; Sesana et al. 2004; Kelley et al. 2017; Mingarelli et al. 2017) detectable by Pulsar Timing Array campaigns (Verbiest et al. 2016) and future spaced-based observations from the Laser Interferometer Space Antenna (Amaro-Seoane et al. 2017). However, SMBH binaries in realistic astrophysical environments can stall on parsec-scale orbits, resulting in merger timescales that exceed the age of the universe. An intruding third SMBH has been shown to significantly shorten coalescence timescales (Ryu et al. 2018) and can also result in slingshot ejections of one of the black holes from the host, resulting in either ejected or wandering SMBHs (e.g., Hoffman \& Loeb 2007; Bonetti et al. 2018, 2019). Triple mergers are therefore of particular interest, because recent cosmological simulations predict that $16 \%$ of binary SMBHs resulting from major mergers will undergo an interaction with a third SMBH prior to coalescing (Kelley et al. 2017). Triple AGN-hosting mergers are exceedingly rare, with only a handful of triple AGN candidates in mergers reported in the literature (Liu et al. 2011; Koss et al. 2012; Kalfountzou et al. 2017) so far. ${ }^{11,12,13}$

In this work we present a suite of multiwavelength observations providing convincing evidence for a triple AGN in the merging system, SDSS J0849+1114. SDSS J0849+1114 is a late-stage merger at a distance of $\sim 350 \mathrm{Mpc}(z=0.077)$ comprising three interacting galaxies, with nuclear pair separations of 2 "! 3 (3.4 kpc, Galaxy 1 - Galaxy 2), 3"!6 (5.3 kpc, Galaxy 1 - Galaxy 3), and 5!"0 (7.3 kpc, Galaxy 2 - Galaxy 3) based upon archival (PI: X. Liu) Hubble Space Telescope (HST) imaging data (Figure 1). Morphologically, the system exhibits strong tidal features indicative of an advanced merger. With an integrated $8-1000 \mu \mathrm{m}$ infrared luminosity of $\log \left(L_{\mathrm{IR}} / L_{\odot}\right)=$ $11.43 \pm 0.03$, this merger falls within the class of luminous infrared galaxies. Based on the all-sky WISE, it displays a strong mid-infrared continuum with colors $(W 1[3.4 \mu \mathrm{m}]-$ $W 2[4.6 \mu \mathrm{m}]=1.69)$ often associated with powerful AGN (Stern et al. 2012), and was therefore included in our previous dual AGN sample. As reported in Pfeifle et al. (2019), SDSS J0849+1114 exhibited three nuclear X-ray sources with luminosities in excess of that expected by star formation contributions, and a high ionization [Si VI] coronal emission line was identified

\footnotetext{
${ }^{10}$ In Pfeifle et al. (2019), two systems were reported as having triple nuclear X-ray sources: SDSS J0849+1114 and SDSS J1306+0735. While the morphology of SDSS J0849+1114 is easily discerned, it is still uncleardespite its three X-ray sources-if SDSS J1306+0735 is a triple merger; a separate investigation is needed to better characterize the latter system.

${ }^{11}$ Barth et al. (2008) classified NGC 3341 as a triple AGN candidate based upon optical spectroscopic line ratios, but Bianchi et al. (2013) suggest it is unlikely that two out of the three galaxies host AGNs based upon their followup multiwavelength analysis.

12 Schawinski et al. (2011) reported the detection of a triple AGN candidate, but - as stated by the authors-given its high redshift, it is unclear if this system resides in a clumpy galaxy where the black holes are forming in situ, or if it is a merging system.

${ }^{13}$ Deane et al. (2014) reported the detection of a triple AGN in the system SDSS J1502+1115 based on 1.7 and $5 \mathrm{GHz}$ observations from VLBI, however higher resolution follow-up observations presented in Wrobel et al. (2014) suggest that this system more likely hosts a dual AGN where one of the AGNs exhibits radio hotspots.
}

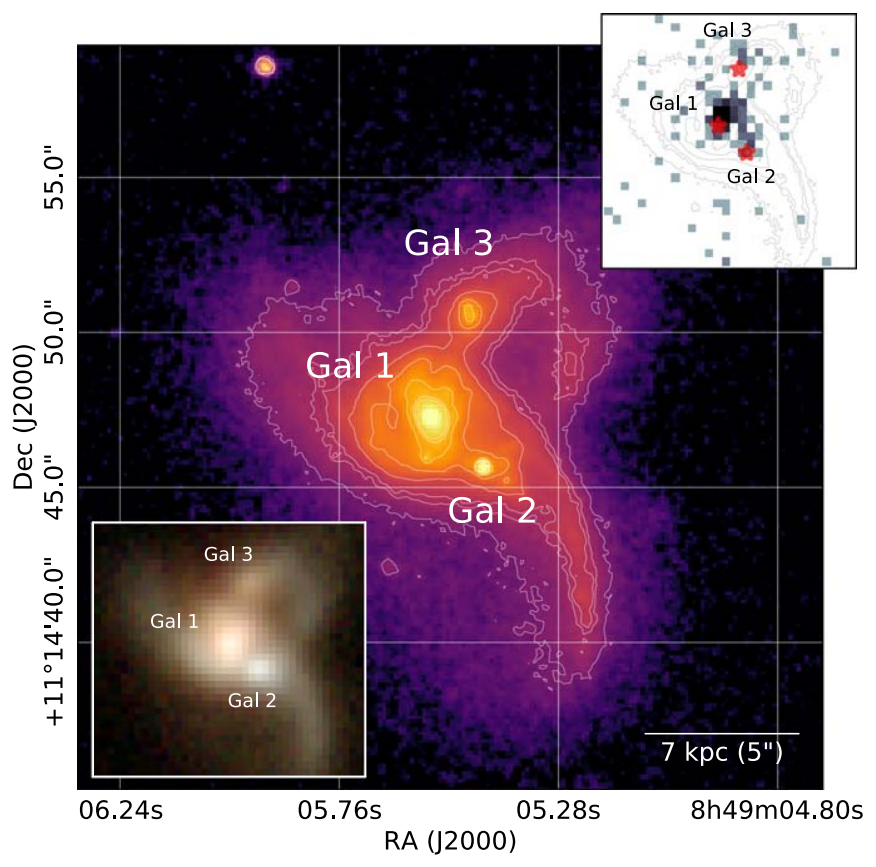

Figure 1. This figure shows the archival HST WFC3 F105W image in the main figure and the combined Chandra $0.3-8 \mathrm{keV}$ X-ray image in the top right corner, with HST contours overlaid on each. The bottom left corner displays the lower resolution SDSS tricolor image. We mark the approximate position of each of the three nuclei on the Chandra image with red stars.

in the near-infrared spectrum of Galaxy 1. Furthermore, the system exhibited signs of high absorption along the line of sight based on its X-ray spectral properties.

Interestingly, this object was also selected independently in a catalog of optically selected AGN pairs in Liu et al. (2011), and was identified as a triple AGN candidate. We combine the archival Chandra data from this program with our own in this analysis.

In this manuscript, we present new NUSTAR hard X-ray observations and X-ray spectral fitting results, new LBT optical spectroscopic observations along with archival SDSS optical spectra, we refine the source statistics using the combined Chandra data sets, and we show for the first time the near-IR spectra for all three galaxy nuclei. The manuscript is laid out as follows: In Section 2 we provide details on the observations and data reduction steps. In Section 3 we discuss the results obtained from the X-ray, near-IR, and optical spectroscopic observations. In Section 4 we explore alternative scenarios for the observed data and discuss this target in the broader context of our mid-infrared selection technique. In Section 5 we summarize our conclusions. Throughout this paper we adopt $H_{0}=70 \mathrm{~km} \mathrm{~s}^{-1} \mathrm{Mpc}^{-1}, \Omega_{M}=0.3$, and $\Omega_{\Lambda}=0.7$.

\section{Observations and Data Reduction}

During our original dual AGN campaign, we obtained Chandra X-ray observations and near-infrared spectroscopy from the LBT of SDSS J0849+1114. As part of a follow-up effort we also obtained hard X-ray NuSTAR observations and longslit optical spectroscopic observations from the LBT. $^{14}$ During this analysis, we also retrieved the archival HST and

\footnotetext{
${ }^{14}$ For our LBT spectroscopic observations, we were careful to obtain observations in optimal seeing conditions $\left(<1^{\prime \prime}\right)$ and extracted the spectra in apertures small enough ( $1^{\prime \prime}$, see Section 2.4) to ensure measurements are restricted to each respective nucleus.
} 
Chandra observations. The Chandra data were reduced using the same procedure outlined in-depth in Pfeifle et al. (2019). Here we describe all other observations.

\subsection{NuSTAR Imaging Observations}

The $31 \mathrm{ks}$ NUSTAR observation was taken on 2017 March 1 (PI: Satyapal) and processed using the NuSTAR Data Analysis Software (NUSTARDAS). To obtain level 2 data products, we ran NUPIPELINE version 0.4.6 with the latest CALDB and clock correction update files. Due to the moderate angular resolution of NUSTAR (FWHM 18"), we used a 30" radius aperture for source extraction and a $30^{\prime \prime}$ aperture for background extraction in the immediate vicinity of the source region. We then created the level three data products using NUPRODUCTS. The spectra generated for FPMA and FPMB were then grouped by 1 count per bin, due to the low number of counts in the spectra, using GRPPHA.

\subsection{Chandra and NuSTAR Spectral Analysis}

All simulations and X-ray spectral fitting for the Chandra and NUSTAR observations were conducted using the XSPEC (Arnaud 1996) X-ray spectral fitting package version 12.9.1 with Cash statistics (C-Stat; Cash 1979). We employed a physically motivated model, BORUS (Baloković et al. 2018), for reprocessed emission along with model components for photoelectric absorption and Compton scattering. This model has four free parameters and is represented by: CONSTANT $\times$ PHABS(BORUSO2 + ZPHABS $\times$ CABS $\times$ CUTOFFPL+CONSTANT $\times$ CUTOFFPL).

Due to the difference in the angular resolution limits of Chandra (PSF FWHM 0..5) and NuSTAR, any hard X-ray photons from the three sources would be confused within the NUSTAR extraction aperture. Before any simultaneous fitting of the observations was performed we first attempted to deconvolve these signals. We began by simulating the 3-24 keV spectra of the three Chandra sources using the BORUS model described above (with $\Gamma=1.9$ ), in each case adjusting the normalization such that the model returned the same $2-10 \mathrm{keV}$ fluxes. We simulated the spectra for a range of column densities $\left(22.0 \leqslant \log \left(N_{\mathrm{H}} / \mathrm{cm}^{-2}\right)\right.$ $\leqslant 25.0$ in increments of $\left.\log \left(N_{\mathrm{H}} / \mathrm{cm}^{-2}\right)=0.5\right)$ and then derived the associated 3-24 keV fluxes. We then fit the NuSTAR spectra with the same BORUS model; based upon the derived NuSTAR flux and the simulated fluxes of the three Chandra sources, it appeared that only Galaxy 1 contributes appreciably to the observed NUSTAR flux. We therefore fit the NUSTAR data simultaneously with the Chandra spectrum of the Galaxy 1 source, only. It is not possible to constrain whether the two remaining AGN are Compton-thick, and it is still possible that they contribute a nonnegligible fraction of the NuSTAR flux.

\subsection{LBT Near-infrared Spectroscopic Observations}

Observations were obtained using LUCI-2 for Galaxies 1 and 2 on 2018 January 30 simultaneously (total of $1440 \mathrm{~s}$ ) using a single slit position with a position angle (PA) of $49^{\circ} .4$ and for Galaxy 3 on 2018 January 3 (total of 2400 s) using the same PA. All observations used a 1."5 wide slit and the low-resolution G200 grating with HKspec filter. This provides a wavelength coverage of $1.46 \mu \mathrm{m}<\lambda<2.34 \mu \mathrm{m}$ and $R \simeq 570-920$. The seeing was $<1^{\prime \prime}$ for all observations. The data were reduced using customized IRAF scripts for flat-fielding, wavelength calibration, and rectification of the spectra. Galaxies 1 and 2 were extracted in a 3 pixel ( 0 ." 75$)$ wide slit and a 4.8 pixel (1". 2$)$ wide slit for Galaxy 3. The spatial apertures were selected to be larger than the seeing to maximize signal-to-noise. Telluric corrections and flux calibration was performed on each galaxy using Version 4.0 of Xtellcor (Vacca et al. 2003).

\subsection{LBT Optical Spectroscopic Observations}

Binocular Multi-Object Double Spectrograph (MODS) observations of Galaxies 1 and 2 were obtained simultaneously on 2018 November 28 using a single slit position with PA $=230^{\circ}$. MODS-1 was configured with the dual grating (blue and red channels) and MODS-2 configured with the blue channel only. Observations were obtained with a 1"! 2 wide slit and a total of $3600 \mathrm{~s}$ for the red channel and $7200 \mathrm{~s}$ combined for the two blue channels. The average seeing during the observations was $<1^{\prime \prime}$. The data were reduced first using version 2.04 of the modsCCDRed python scripts and then flat-fielded, wavelength calibrated, rectified, and flux-calibrated using customized IRAF scripts. A $1 D$ spectrum for each galaxy was extracted in a $1^{\prime \prime}$ wide spatial diameter based on the same criteria as the near-IR spectra.

\subsection{LBT Optical Spectral Fitting to Determine Fluxes and Stellar Velocity Dispersion}

In order to measure accurate line fluxes, it is necessary to account for stellar absorption, which primarily affects the Balmer lines. The Penalized Pixel-Fitting method (pPXF; Cappellari 2017) is an algorithm developed to extract stellar kinematics using a maximum penalized likelihood approach to match stellar spectral templates to absorption features in galaxy spectra. A large $(>150)$ number of stellar templates of various spectral type were chosen from the Indo-US Library of Coudé Feed Stellar Spectra (Valdes et al. 2004) in order to decrease the possibility of template mismatch. To ensure that the Balmer absorption is adequately constrained, we model the stellar population using the full spectrum (4000-7500 ̊) with pPXF. After subtracting the best-fitting stellar population model (red, see Figure 2$)^{15}$ from the continuum, the residual emission lines were fit using Gaussian models. Broadened wing components, which can be indicative of high-velocity gas outflows, are present in narrow lines of all three objects, and thus an additional Gaussian component is added (see Figure 2 for examples of emission line decompositions). The presence of $\mathrm{Fe}$ emission can also affect measurements of the stellar population and [O III] $\lambda \lambda 4959,5007$ doublet, and therefore we include the Fe II template from Véron-Cetty et al. (2004) and Fe I lines from the NIST Atomic Spectra Database in our fits. Fitting was performed using a custom maximum-likelihood routine implemented in Python (Sexton et al. 2019), which uses the affine-invariant MCMC ensemble sampler emcee (ForemanMackey et al. 2013). Line fluxes are determined from the bestfit Gaussian models of the emission lines. Stellar-velocity dispersions were fit using a similar method described, except we fit the region from 4400 to $5800 \AA$, which includes the $\mathrm{Mg} \mathrm{Ib}$ region used to estimate stellar velocity dispersion. The [O III] doublet is best fit by a combination of two Gaussian components. The line widths from the [O III] doublet fit are then used to constrain the widths of the rest of the emission lines (Rodríguez Zaurín et al. 2013), so careful modeling of their profiles is necessary. The fitting routine can actually

\footnotetext{
15 Figure 8 in the Appendix illustrates the full dynamic range of the optical spectra.
} 

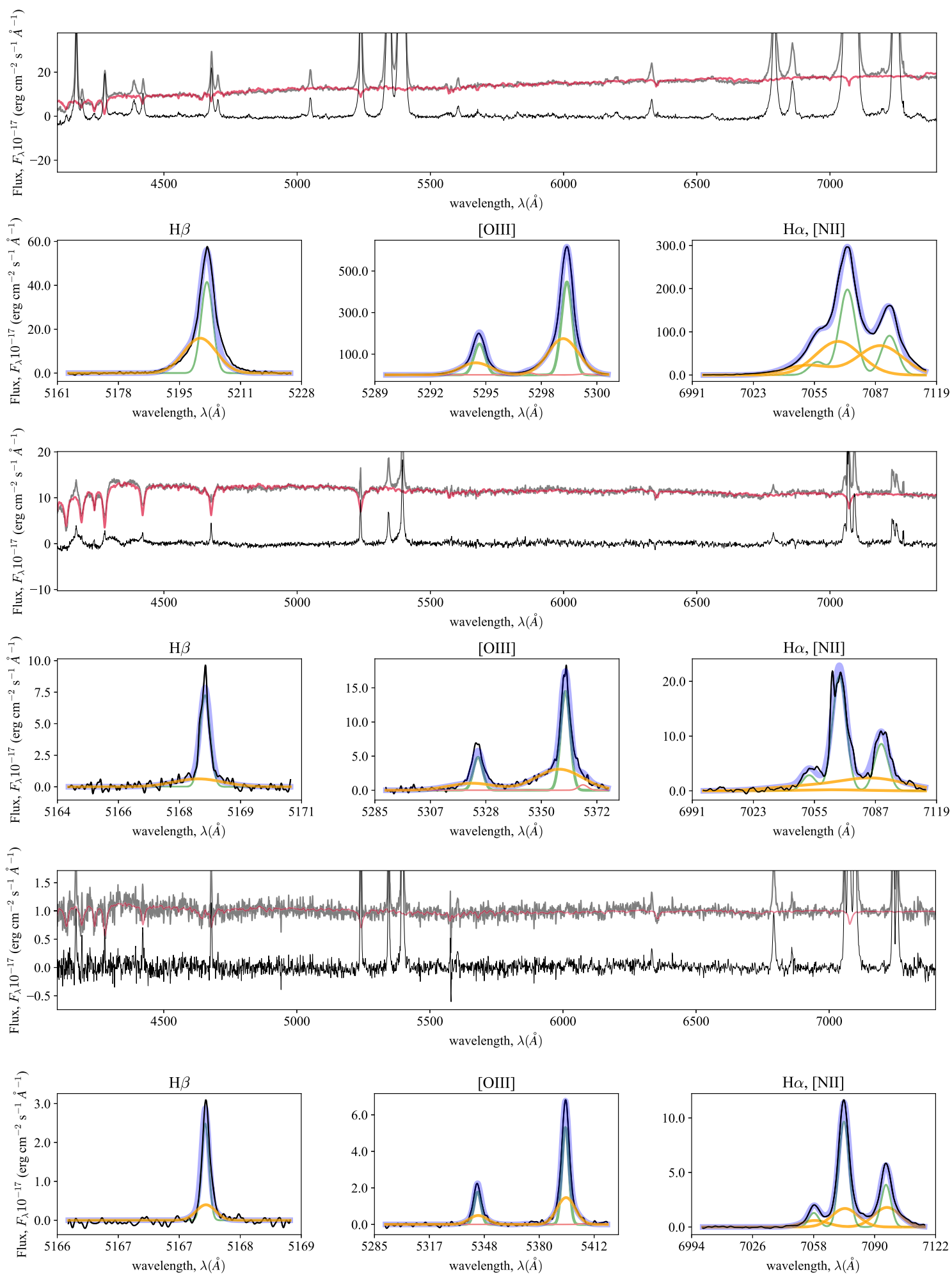

Figure 2. Optical spectra of the three nuclei within SDSS J0849+1114, where rows one (Galaxy 1), three (Galaxy 2), and five (Galaxy 3) show the full (observed frame) optical spectrum of each nucleus, while rows two, four, and six show specific examples of emission line decompositions for Galaxies 1,2, and 3. The spectra for Galaxies 1 and 2 were obtained with the MODS on LBT while the archival spectrum for Galaxy 3 was obtained from SDSS. Figure 8 in the Appendix illustrates the full dynamic range of these spectra; here we show the spectral region used for pPXF analysis to aid the reader in examining the stellar population subtraction. The spectra were fit using the procedure outlined in Section 2.5; the light gray spectrum in rows 1,3, and 5 shows the full observed spectrum, the red line illustrates the spectrum of the best-fitting stellar population, and the black spectrum of each panel shows the resulting spectrum after subtracting the stellar population model. The sets of panels illustrating the emission line decompositions are color-coded, where the orange shading represents the broad line component, the green shading represents the narrow line component, and the blue shading represents the combined (full) emission line. The solid black line in each decomposition panel shows the observed emission line from the spectrum after the stellar continuum subtraction. The thin red line in each [O III] $\lambda 5007$ decomposition panel shows the contaminating Fe I $\lambda 4985, \lambda 4986, \lambda 5016$ emission. 
Table 1

Chandra X-Ray Source Statistics

\begin{tabular}{|c|c|c|c|c|c|c|c|c|c|}
\hline Galaxy & $\left(10^{20} \mathrm{~cm}_{\mathrm{H}}^{N^{-2}}\right)$ & $\alpha_{\chi}$ & $\delta_{x}$ & $\begin{array}{c}\text { Counts } \\
0.3-8 \mathrm{keV}\end{array}$ & $\begin{array}{c}\text { Counts } \\
0.3-2 \mathrm{keV}\end{array}$ & $\begin{array}{l}\text { Counts } \\
2-8 \mathrm{keV}\end{array}$ & HR & $\sigma$ & $\log \left(P_{B}\right)$ \\
\hline 1 & 3.80 & $8^{\mathrm{h}} 49^{\mathrm{m}} 05^{\mathrm{s}} .529$ & $+11 \operatorname{deg} 14^{\prime} 47^{\prime \prime} 876$ & $196 \pm 14$ & $112 \pm 11$ & $84 \pm 9$ & -0.14 & $13.8 \sigma$ & -371.6 \\
\hline 2 & 3.80 & $8^{\mathrm{h}} 49^{\mathrm{m}} 05^{\mathrm{s}} \cdot 381$ & +11deg14'45".747 & $15_{-4}^{+5}$ & $13_{-2}^{+4}$ & $2_{-1}^{+3}$ & -0.76 & $3.5 \sigma$ & -17.3 \\
\hline
\end{tabular}

Note. Column 1: X-ray source and galaxy nucleus designation. Column 2: Galactic hydrogen column density along the line of sight. Column 3-4: right ascension (R.A.) and declination (decl.) of the source. Column 5-7: X-ray counts detected in the full, soft, and hard X-ray bands for the combined 2013 and 2016 Chandra observations. Note that these are not background-subtracted counts, but formal statistical significances of the sources are derived from background-subtracted statistics. Values are rounded to closest integer. Column 8: hardness ratio, given by the formula $(\mathrm{H}-\mathrm{S}) /(\mathrm{H}+\mathrm{S})$, where $\mathrm{H}$ and $\mathrm{S}$ refer to the Chandra hard and soft energy bands. Column 9: formal source statistical significance, derived from background-subtracted source statistics. Column 10: logarithm of the binomial source probability, which is a metric for source statistical significance more appropriate for the low-count regime. Note: sources which meet a threshold of $\log \left(P_{B}\right)<-2.7$ are considered to be true sources, and it is highly unlikely that these are the result of spurious background activity (Lansbury et al. 2014); we used this statistical metric for our dual sample in both Satyapal et al. (2017) and Pfeifle et al. (2019).

overestimate the flux and width of the broad component of the [O III] $]$ 5007 emission line due to contaminating flux from the Fe I $\lambda 4985, \lambda 4986, \lambda 5016$ emission lines. To address this contamination, we model the $\mathrm{Fe}$ lines with independent amplitudes and with widths equal to that of the narrow component of [O III] (Manzano-King et al. 2019).

\section{Results}

\subsection{Chandra/ACIS-S Imaging Results}

We combined our 2016 observation with an archival 2013 observation (PI: X. Liu), and assessed source significance using the binomial no-source probability statistic $\left(P_{B}\right)$ (Lansbury et al. 2014), which is suitable for the low-count regime; we point the reader to Pfeifle et al. (2019) for further discussion. The Chandra source statistics are reported in Table 1. The Galaxy 1, 2 , and 3 sources were detected with a statistical significance of $13.8 \sigma\left[\log \left(P_{B}\right)=-371.6\right], 3.5 \sigma\left[\log \left(P_{B}\right)=-17.3\right]$, and $2.9 \sigma$ $\left[\log \left(P_{B}\right)=-12.0\right]$, and based upon the derived $P_{B}$ values it is highly unlikely that these sources are the result of spurious background activity. ${ }^{16}$ We used the CIAO MODELFLUX package to uniformly estimate the hard $2-10 \mathrm{keV}$ (rest frame) luminosities for the sources, assuming $\Gamma=1.9$ and correcting for only Galactic $N_{\mathrm{H}}$ along the line of sight. Galaxies 1,2, and 3 exhibit $2-10 \mathrm{keV}$ luminosities of $2.37 \pm 0.17 \times 10^{41} \mathrm{erg} \mathrm{s}^{-1}, 2.4_{-0.7}^{+0.8} \times$ $10^{40} \mathrm{erg} \mathrm{s}^{-1}, 1.5_{-0.5}^{+0.6} \times 10^{40} \mathrm{erg} \mathrm{s}^{-1}$ respectively.

\subsection{Chandra/ACIS-S and NuSTAR Spectral Analysis Results}

Simultaneously fitting the Chandra and NuSTAR data for Galaxy 1 (see Figure 3), we find an absorbing column of $\log \left(N_{\mathrm{H}}\right.$ $\left./ \mathrm{cm}^{-2}\right)=23.9_{-0.2}^{+0.2}$, photon index $\Gamma=1.4_{\mathrm{NC}}^{+0.3}$, and a scattered fraction $f_{S}=11.7_{-6.4}^{+22.1} \% .{ }^{17}$ Correcting for the intrinsic absorption, we find an unabsorbed $2-10 \mathrm{keV}$ luminosity of $4.6_{-0.6}^{+0.6} \times$ $10^{42} \mathrm{erg} \mathrm{s}^{-1}$. We also report the NUSTAR source statistics in Table 2. To measure the equivalent width of the $\mathrm{Fe} \mathrm{K} \alpha$ line, we repeated the fitting process with the phenomenological model from Pfeifle et al. (2019), which includes a Gaussian emission line component to account for the iron line fluorescent emission. Due to the signal-to-noise ratio of our X-ray data,

\footnotetext{
16 These significance values differ slightly from those reported in Pfeifle et al. (2019) due to the fact that here we quote the statistical significance derived from the combined Chandra statistics, whereas previously the statistical significance was derived from only the 2016 Chandra data set.

${ }^{17} \mathrm{NC}=$ Not constrained.
}

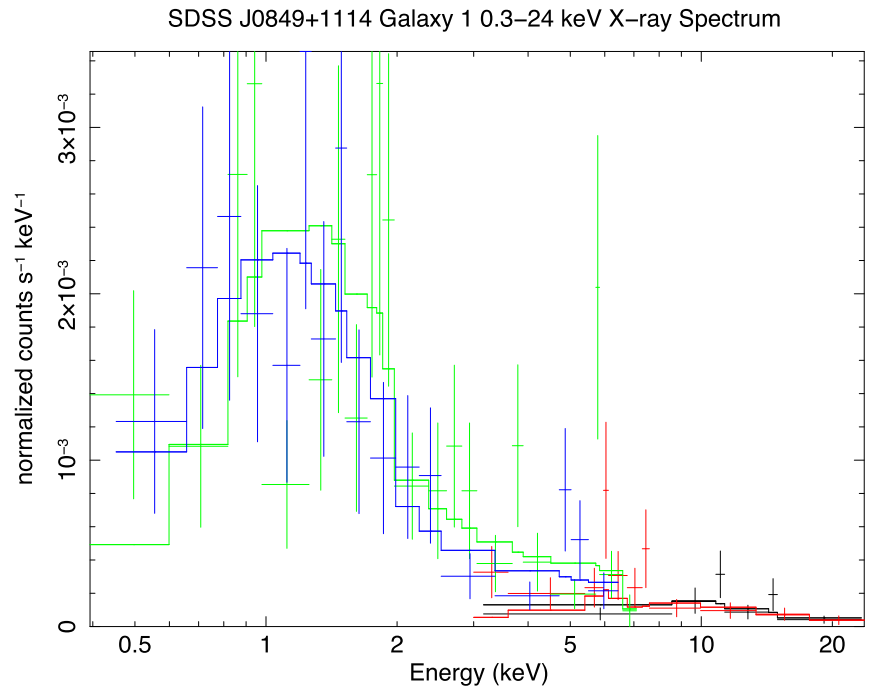

Figure 3. This diagram depicts the NUSTAR 3-24 keV (black: FPMA, red FPMB; PI: Satyapal) and two Chandra $0.3-8 \mathrm{keV}$ spectra (green: PI, Satyapal; blue: PI, Liu) along with the best-fitting model to the data. Fitting with the Borus reprocessed emission model, along with components for Comptonscattering and reflected emission, reveals that the Galaxy 1 source is a heavily obscured AGN.

we conservatively quote here only an upper limit for the $\mathrm{Fe} \mathrm{K} \alpha$ emission. We find an equivalent width upper limit of $\sim 0.6 \mathrm{keV}$ for the $\mathrm{Fe} \mathrm{K} \alpha$ line, which is consistent with the high absorbing column along the line of sight (Brightman \& Nandra 2011).

While we do find different values for the spectral parameters here than those reported in Pfeifle et al. (2019), this is not entirely surprising; in this manuscript we are fitting both sets of Chandra and NuSTAR observations simultaneously, and it is well known that NUSTAR observations aid significantly in constraining the values for spectral parameters such as the photon index, $N_{\mathrm{H}}$, and the equivalent width of the $\mathrm{Fe} \mathrm{K} \alpha$ line (Marchesi et al. 2018), in some cases leading to refined values that are significantly different from those found with data in the $2-10 \mathrm{keV}$ energy range.

As we discussed in Section 2.2, it is not possible to constrain whether the two remaining AGNs are Compton-thick, and it is still possible they contribute appreciably to the observed hard $\mathrm{X}$-ray flux because they would fall within the NUSTAR extraction aperture. In any case, this does not affect our X-ray spectral fitting results; the results are consistent with the picture of mergers 

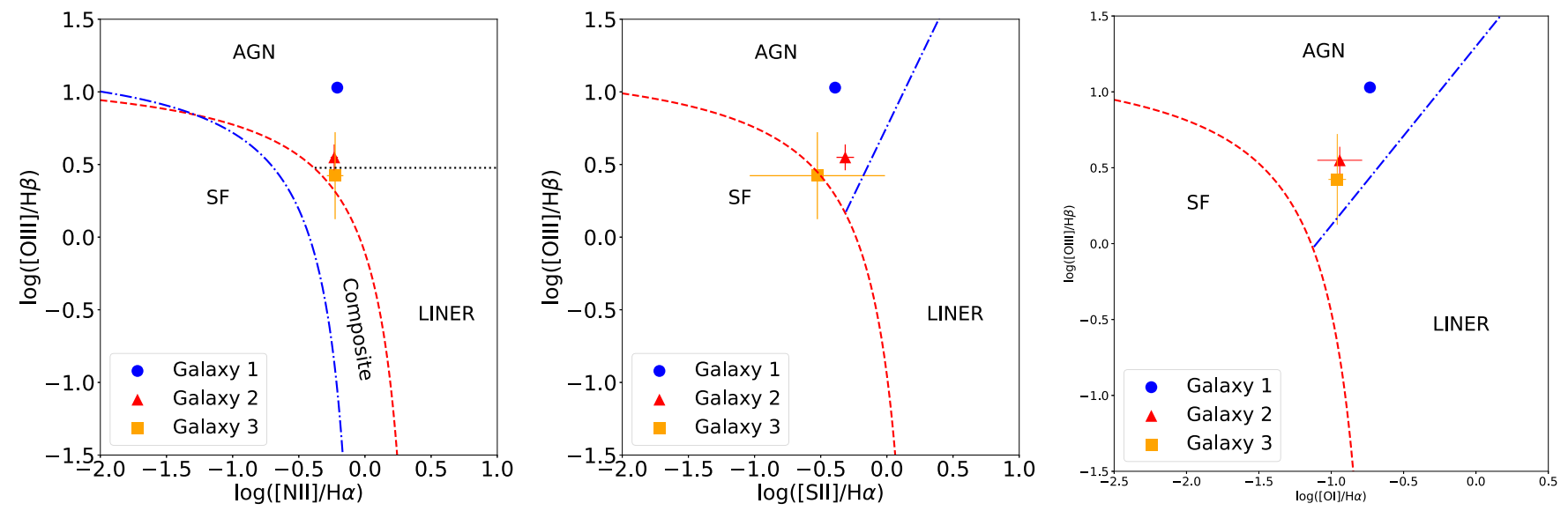

Figure 4. Three BPT optical spectroscopic line ratio diagrams, based on the $[\mathrm{O}$ III $] \lambda 5006 / \mathrm{H} \beta$ to $[\mathrm{N}$ II] $/ \mathrm{H} \alpha$, [S II] $] \lambda \lambda 6717,6733 / \mathrm{H} \alpha$ doublet, and $[\mathrm{O}$ I] $\lambda 6302 / \mathrm{H} \alpha$ emission line ratios. Left: the red (dashed), blue (dashed-dotted), and black (dotted) lines represent the Kewley et al. (2001), Kauffmann et al. (2003), and Ho et al. (1997) demarcations, respectively, which separate AGN, star-forming, and LINER populations. Center and right: the red (dashed) and blue (dashed-dotted) lines represent the Kewley et al. (2001) and Kewley et al. (2006) demarcations, which separate H II regions, Seyferts, and LINERs. All three nuclei display AGN-like line ratios. The [S II] and [O I] ratios are inconsistent with shock-driven photoionization (Dopita \& Sutherland 1995), which would typically produce line ratios in the lower right (LINER) corners of the middle and right diagrams. The logarithmic emission line ratios plotted above are listed in Table 3.

Table 2

NUSTAR X-Ray Source Statistics

\begin{tabular}{|c|c|c|c|c|c|c|}
\hline Camera & $N_{\mathrm{H}}$ & $\alpha_{\chi}$ & $\overline{\delta_{\chi}}$ & $\begin{array}{c}\text { Counts } \\
3-78 \mathrm{keV}\end{array}$ & $\begin{array}{c}\text { Counts } \\
3-8 \mathrm{keV}\end{array}$ & $\begin{array}{c}\text { Counts } \\
8-24 \mathrm{keV}\end{array}$ \\
\hline FPMA & 3.80 & $8^{\mathrm{h}} 49^{\mathrm{m}} 05^{\mathrm{s}} .510$ & $+11 \operatorname{deg} 14^{\prime} 47$ !" $^{\prime \prime} 60$ & $141 \pm 12$ & $20 \pm 4$ & $63 \pm 8$ \\
\hline FPMB & 3.80 & $8^{\mathrm{h}} 49^{\mathrm{m}} 05^{\mathrm{s}} .510$ & $+11 \operatorname{deg} 14^{\prime} 47$ !' $^{\prime \prime} 60$ & $149 \pm 12$ & $49 \pm 7$ & $59 \pm 8$ \\
\hline
\end{tabular}

Note. Column 1: NUSTAR camera. Column 2: Galactic hydrogen column density along the line of sight. Column 3-4: R.A. and decl. coordinates of the source apertures. Column 5: X-ray counts in the 3-78 keV NuSTAR band. Column 6: X-ray counts in the 3-8 keV NuSTAR band. Column 7: X-ray counts in the 8-24 keV NuSTAR band.

Table 3

LBT and SDSS Spectroscopic Results

\begin{tabular}{lcccccc}
\hline \hline Galaxy & $\log ([\mathrm{O}$ III] $] \lambda 5006 / \mathrm{H} \beta)$ & $\log ([\mathrm{N} \mathrm{II}] / \mathrm{H} \alpha)$ & $\log ([\mathrm{S} \mathrm{II}] \lambda \lambda 6717,6733 / \mathrm{H} \alpha)$ & $\log ([\mathrm{O} \mathrm{I}] \lambda 6302 / \mathrm{H} \alpha)$ & $\begin{array}{c}\sigma_{*} \\
\left(\mathrm{~km} \mathrm{~s}^{-1}\right)\end{array}$ \\
\hline 1 & $1.029 \pm 0.009$ & $-0.209 \pm 0.003$ & $-0.39 \pm 0.01$ & $-0.733 \pm 0.006$ & $180 \pm 9$ \\
2 & $0.55 \pm 0.09$ & $-0.232 \pm 0.03$ & $-0.31 \pm 0.07$ & $-0.9 \pm 0.2$ & $149 \pm 11$ \\
3 & $0.4 \pm 0.3$ & $-0.23 \pm 0.06$ & $-0.5 \pm 0.5$ & $-0.96 \pm 0.06$ & $91 \pm 26$ \\
\hline
\end{tabular}

Note. Column 1: galaxy nucleus designation. Columns 2-5: logarithmic optical emission line ratios for each nucleus, calculated using the derived optical emission line fluxes. See Section 2.5 for more details on how these line fluxes were derived from the optical spectra. Column 6: Stellar velocity dispersion. Column 7: gas velocity dispersion. The large uncertainties in gas and velocity dispersions for Galaxy 3 are due to the lower signal-to-noise of SDSS spectra compared to LBT-obtained spectra.

hosting obscured AGNs, as found by previous studies (Kocevski et al. 2015; Ricci et al. 2017, 2016; Donley et al. 2018; Goulding et al. 2018; Koss et al. 2018; Lanzuisi et al. 2018).

\subsection{LBT Spectroscopic Results}

To determine the nature of the photoionizing sources observed in the LBT optical spectroscopy, we employ "Baldwin, Phillips, and Terlevich" (BPT) optical spectroscopic diagnostic diagrams, shown in Figure 4. The optical emission in each nucleus is consistent with AGN photoionization, complementing the X-ray and near-IR observations, and is consistent with the presence of a triple AGN in this merger. We list the logarithmic emission line ratios in Table 3.

Following the procedure outlined in Section 2.5, we derived stellar and gas velocity dispersions for each nucleus, which we report in Table 3. Gas velocity dispersions were measured from the narrow component of the $[\mathrm{O}$ III $] \lambda 5007$ emission line while the stellar velocity dispersions were measured by fitting the $\mathrm{Mg}$ Ib region in the spectral range $4400-5800 \AA$. We find very similar values for the stellar and gas velocity dispersions within each respective nucleus (see Columns 6-7 in Table 3), but these values differ between the three nuclei; these similarities further suggest that each of the three regions are more-or-less dynamically independent- that is, the gas and stellar components are well-coupled-especially for Galaxies 1 and $3 .^{18}$

We observe strong blue-wing components [O III] $\lambda 5007$ emission lines in Galaxy 1 and 2 with FWHMs on the order of $\sim 1300-1600 \mathrm{~km} \mathrm{~s}^{-1}$, which is suggestive of strong galactic-scale

\footnotetext{
${ }^{18}$ We caution the reader that the interpretation of velocity dispersions is highly uncertain in merging systems (Stickley \& Canalizo 2014). We list these dispersions here simply to support the scenario of three kinematically distinct nuclei.
} 


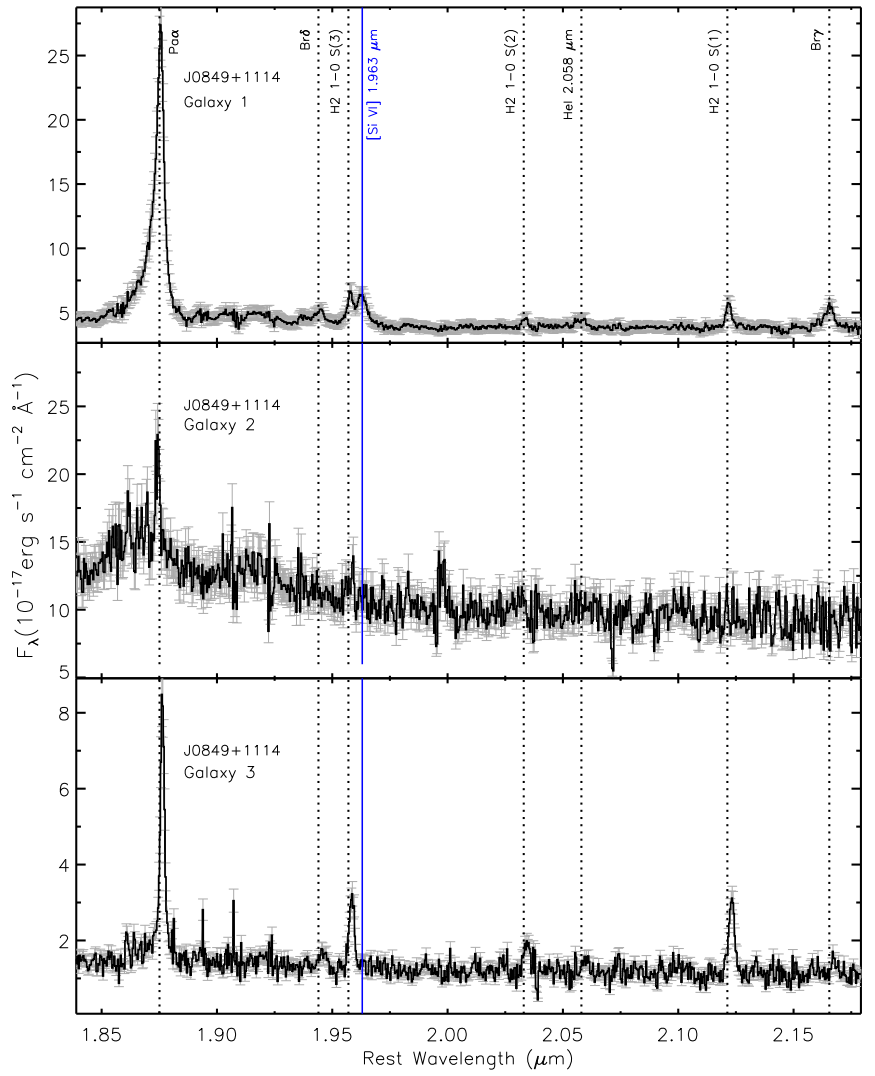

Figure 5. These three panels show the near-IR $K$-band spectra for the three nuclei in SDSS J0849+1114 obtained from the LBT, where the top, middle, and bottom panels correspond to the spectra for Galaxy 1,2, and 3. Dotted black lines indicate hydrogen and helium line emission, while the blue line indicates the wavelength corresponding to the [Si VI] $1.967 \mu \mathrm{m}$ high ionization coronal emission line. One [Si VI]1.967 $\mu \mathrm{m}$ coronal line is detected in Galaxy $1(\sigma=2.3)$. The detection of a coronal line provides robust confirmation for the AGN in this nucleus.

outflows (Heckman et al. 1981; Nelson \& Whittle 1996; Woo et al. 2016). Galaxy 3 shows a slightly redshifted wing in the [O III] $\lambda 5007$ emission, with an FWHM on the order of $\sim 700 \mathrm{~km}$ $\mathrm{s}^{-1}$. Many of the lower ionization emission lines show broadwing components as well.

The LBT near-IR spectra revealed high excitation [Si VI] (Pfeifle et al. 2019) coronal line emission $\left(f_{\lambda}=1.63 \pm 0.07 \times\right.$ $10^{-15} \mathrm{erg} \mathrm{cm}^{-2} \mathrm{~s}^{-1}$ ) in the nucleus of Galaxy 1, providing robust confirmation for the AGN in that nucleus (see Figure 5). Moreover, the near-infrared spectra of Galaxy 1 and Galaxy 3 reveal broad components (FWHM $\gtrsim 2400 \mathrm{~km} \mathrm{~s}^{-1}$ ) in the $\mathrm{Pa} \alpha$ emission (see Figure 6), significantly in excess of the blueshifted components observed in the [O III] $\lambda 5007$ emission. These detections offer additional evidence for the existence of black hole accretion in these galaxies. We point out that this accretion activity is likely missed in the optical due to large obscuring columns along the line of sight. An exhaustive analysis of the possible outflows observed in the optical spectra is, however, beyond the scope of this paper, but will be featured in a future publication focusing on the environmental impacts of AGNs in mergers.

\section{Discussion and Implications}

While our multiwavelength observations point to each nucleus hosting an AGN, we considered alternative scenarios that might explain the observed characteristics of this system.

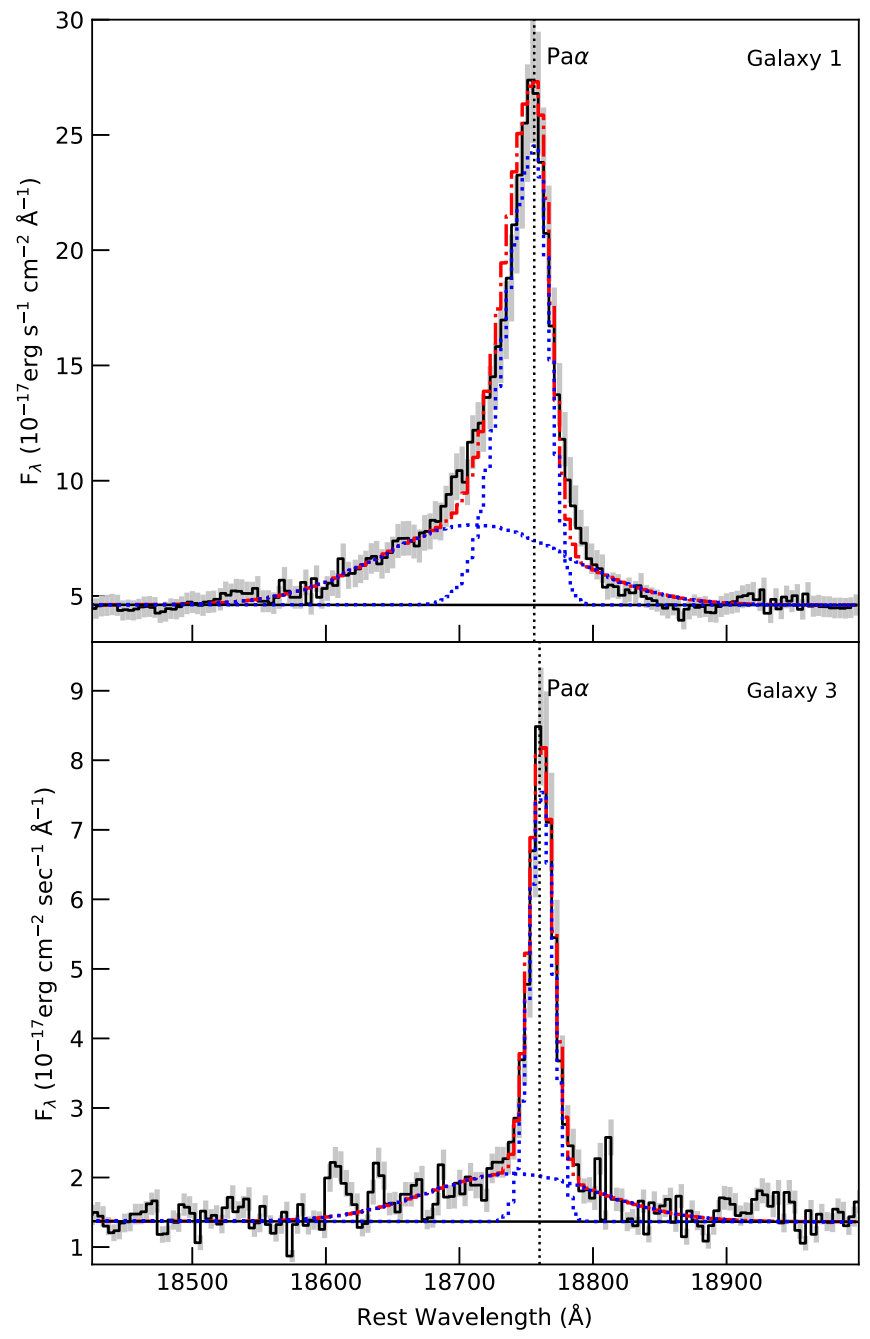

Figure 6. These panels show profile fits for the Pa $\alpha$ near-infrared emission in the spectra of Galaxies 1 and 3. These broad components (FWHM $\gtrsim 2400 \mathrm{~km}$ $\mathrm{s}^{-1}$ ) in the $\mathrm{Pa} \alpha$ emission are significantly in excess of the blueshifted components observed in the [O III] $\lambda 5007$ emission, consistent with a broad line region in these two galaxies. Note that the quality of the spectra in Galaxy 2 was insufficient for an emission line decomposition.

In order to rule out star formation as an alternative explanation for the observed X-ray emission, we calculated star formation rates (SFRs) and possible X-ray contributions from a population of X-ray binaries using the $\mathrm{Pa} \alpha$ emission observed in the LBT near-IR spectra. We refer the reader to Satyapal et al. (2017) and Pfeifle et al. (2019) for a discussion of the SFR and XRB contribution calculations. With this calculation, we estimate an upper limit for the star formation activity in the merger nuclei; in reality, because the nuclei all exhibit BPT AGN-like optical spectroscopic line ratios, some fraction of the $\mathrm{Pa} \alpha$ emission is in fact attributable to the AGNs and therefore the true SFR, and hence stellar contribution to the X-rays, is much lower.

We obtained SFRs of $13.16 M_{\odot} \mathrm{yr}^{-1}, 0.48 M_{\odot} \mathrm{yr}^{-1}$, and $1.79 M_{\odot} \mathrm{yr}^{-1}$ and predicted hard $2-10 \mathrm{keV}$ X-ray luminosities of $2.27 \pm 0.4 \times 10^{40} \mathrm{erg} \mathrm{s}^{-1}, 0.12 \pm 0.11 \times 10^{40} \mathrm{erg} \mathrm{s}^{-1}$, and $0.43 \pm 0.20 \times 10^{40} \mathrm{erg} \mathrm{s}^{-1}$ for Galaxy 1,2 , and 3 , respectively. Contrasting these values with the observed X-ray emission (uncorrected for intrinsic absorption) in Galaxies 1, 2, and 3 (see Section 3.1), there is a clear order of magnitude disparity between the observed and predicted values, strongly suggestive of three AGNs. Once again we note that in reality - due to the 
expected contributions from the AGNs to the $\mathrm{Pa} \alpha$ flux-we are likely overestimating the X-ray contributions from SF. Moreover, we are underestimating the X-ray fluxes of the three sources as the quoted luminosities are not corrected for absorption; as indicated by our joint NUSTAR-Chandra spectral fitting results, any absorption corrections made to the observed $\mathrm{X}$-ray luminosities are expected to be large.

While we have confirmed the AGN in Galaxy 1 via the detection of a near-IR coronal line, Galaxies 2 and 3 require closer examination. If the SFR within Galaxies 2 and 3 were indeed $\sim 30 M_{\odot} \mathrm{yr}^{-1}$ and $\sim 9 M_{\odot} \mathrm{yr}^{-1}$, respectively-a requirement to explain the observed X-ray emission-the optical spectra should be dominated by a very young $(<10 \mathrm{Myr})$ and blue stellar population, when the population of high mass X-ray binaries (HMXBs) is expected to be high (von der Linden et al. 2010). The most luminous source of $X$-ray emission in galaxies arises from HMXBs. ${ }^{19}$ Our near-infrared spectra allow us to also test this hypothesis by constraining the ages of the nuclear stellar populations. Specifically, the equivalent width of hydrogen recombination lines show a steep decline as the most massive stars evolve off the main sequence, causing a simultaneous decrease in the ionizing photon flux and an increase in the $K$-band continuum flux (Leitherer et al. 1999). However, the equivalent width of the $\mathrm{Br}-\gamma$ line (or upper limit in the case of Galaxy 2 and 3), a strong indicator of the starburst age (Leitherer et al. 1999, 2014), suggests that the stellar population in all three galaxies is greater than $\sim 6$ million years (Figure 7), well after the time when the population of HMXBs is expected to drop dramatically (von der Linden et al. 2010).

We also considered the possibility that the optical emission lines are produced by ionizing shocks powered by starburst driven winds or tidal flows (Veilleux et al. 2002; Colina et al. 2005). Shocks are known to arise in mergers and become an increasingly important component of the optical emission lines as a merger progresses (Rich et al. 2011), generating [O III] $/ \mathrm{H} \beta$ and $[\mathrm{N} \mathrm{II}] / \mathrm{H} \alpha$ ratios consistent with AGN when in fact only star formation is present (Allen et al. 2008). However, shock models have shown that when there is a significant contribution from shocks to the ionization, it often results in enhanced [O I] $/ \mathrm{H} \alpha$ and $[\mathrm{S} \mathrm{II}] / \mathrm{H} \alpha$ emission line ratios compared to AGN photoionization models (Dopita \& Sutherland 1995; Allen et al. 2008), which is not seen in any of the three nuclei. Furthermore, detection of three X-ray point sources coincident with the nuclei supports an AGN origin rather than the result of shockheated gas.

Finally, we explored the possibility that there are fewer than three distinct sources of ionization (one located at each galactic nucleus). In particular, given the [O III] luminosities of each nucleus, the typical narrow line region size is expected to be comparable to the nuclear pair separations between Galaxy 1 and Galaxy 2 (Bennert et al. 2006; Hainline et al. 2013, 2014), raising the possibility that only one AGN is ionizing the gas at both locations. However, the detection of three separate luminous X-ray sources at the location of each nucleus makes this scenario unlikely. Furthermore, the velocity offset of the [O III] lines between the two nuclei is consistent with the

\footnotetext{
${ }^{19}$ Ultraluminous X-ray sources (ULXs) can be more luminous than HMXBs however, the vast majority of these objects exhibit unabsorbed $0.2-10 \mathrm{keV}$ luminosities between $10^{39}$ and $10^{40} \mathrm{erg} \mathrm{s}^{-1}$ (Sutton et al. 2012), which fall below the absorbed luminosities of the sources presented here. Additionally, ULXs are not generally associated with mid-infrared AGN colors (Secrest et al. 2015)
}

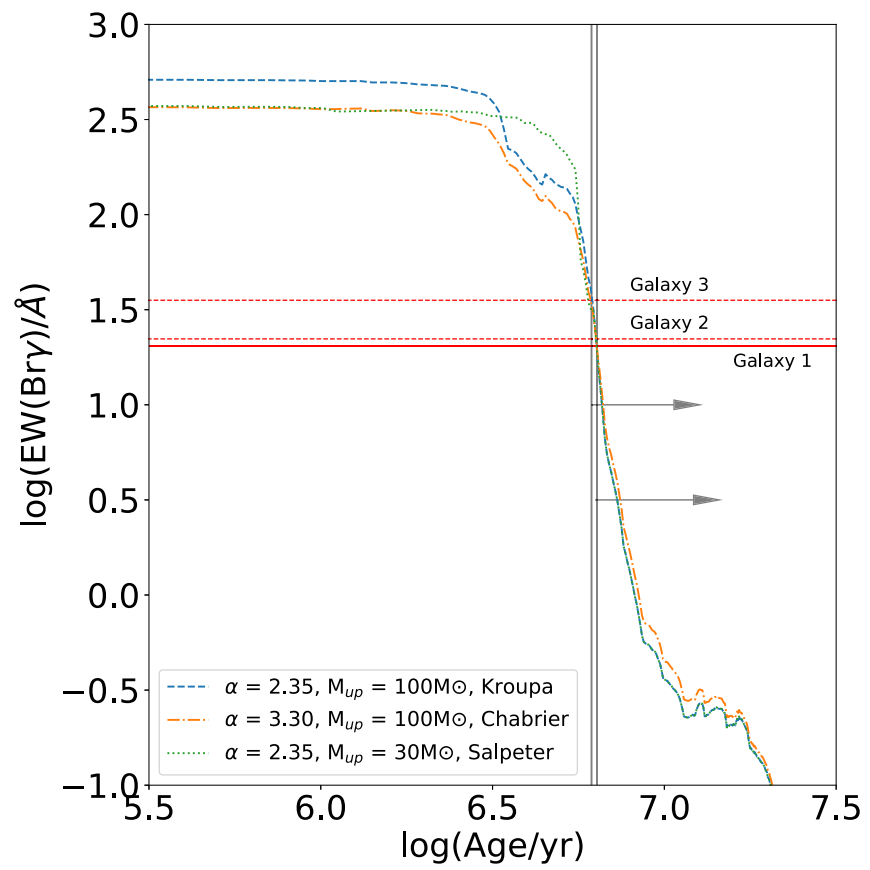

Figure 7. Equivalent width of the $\mathrm{Br} \gamma$ emission line from Starburst 99 starburst models (Leitherer et al. 1999) for three different initial mass functions at solar metallicity. The red horizontal lines represent the observed values measured for each nucleus; we use dotted line styles for Galaxies 2 and 3 to denote that these ages are upper limits. The vertical black lines constrain the ages of the relatively young stellar populations to $\sim 6.1-6.4 \mathrm{Myr}$, beyond the age at which the HMXB population is expected to peak.

velocity offset measured for the $\mathrm{H} \beta$ lines, suggesting a lack of ionization stratification (Zamanov et al. 2002) expected for a single AGN, and therefore also favoring a scenario in which there are two independent sources of ionization. Moreover, each galaxy has unique, well-coupled gas and stellar velocity dispersions, suggesting three kinematically distinct sources consistent with galaxy nuclei.

We note that SDSS J0849+1114 was targeted for multiwavelength follow-up as a result of mid-infrared color preselection (Satyapal et al. 2014, 2017; Pfeifle et al. 2019). While optical preselection strategies (Liu et al. 2011; Comerford et al. 2015) are extensively used in campaigns to identify dual AGNs, these strategies have yielded very few confirmed cases. In fact, very few late-stage galaxy mergers are known to host dual AGNs (see Table 8 from Satyapal et al. 2017), most of which have been discovered serendipitously or through hard X-ray surveys (Koss et al. 2012). While there have been a couple of triplet quasars reported in the literature (Djorgovski et al. 2007; Farina et al. 2013), they are at much larger separations and it is unclear if they are hosted in mergers. A few candidate triple AGNs in mergers have been reported (Liu et al. 2011; Koss et al. 2012; Kalfountzou et al. 2017) but thus far there have been no cases exhibiting triple $\mathrm{X}$-ray point sources and optical narrow line signatures consistent with AGNs. Emission from the nuclei of advanced mergers at these pair separations are expected to be highly obscured (e.g., Kocevski et al. 2015; Ricci et al. 2017; Blecha et al. 2018), making it difficult to identify dual AGNs using traditional techniques. While it is true that SDSS J0849+1114 exhibits optical signatures of AGNs in each nucleus, it is unlikely if all triple AGNs will exhibit such optical spectroscopic signatures. In contrast, mid-infrared preselection has proven an effective strategy in finding dual AGNs and now a triple AGN candidate in 
follow-up multiwavelength studies (Ellison et al. 2017; Satyapal et al. 2017; Pfeifle et al. 2019), suggesting that similar cases to SDSS J0849+1114 can be found in future studies.

\section{Conclusion}

In this investigation, we employed a suite of multiwavelength diagnostics which yield a compelling case for the existence of an AGN triplet in the triple galaxy merger SDSS J0849+1114. This merger, preselected in our dual AGN campaign for follow-up observations based on its WISE mid-infrared colors, was only later realized as a triple AGN candidate (Pfeifle et al. 2019). The nature of SDSS J0849+1114 can be summarized as follows:

1. Chandra revealed three nuclear X-ray point sources which exhibit luminosities in excess of any expected contributions from stellar activity.

2. High excitation [Si VI] coronal line emission was detected in the nucleus of Galaxy 1 via the near-infrared longslit spectra obtained with the LBT. We also find evidence for broad components in the near-IR Pa $\alpha$ emission of Galaxy 1 and 3, with significantly greater widths than those observed in the broad components of the optical [O III] $\lambda 5007$ emission.

3. New longslit optical spectroscopic measurements shown for the first time here for Galaxy 1 and Galaxy 2, along with the archival SDSS spectrum for Galaxy 3, reveal optical spectroscopic emission line ratios consistent with AGN photoionization in all three nuclei.

4. Simultaneous spectral fitting of the Chandra and new NUSTAR observations confirms at least one of the nuclei (Galaxy 1) hosts a heavily absorbed AGN and we further refine our previously reported spectral parameters: $\log \left(N_{\mathrm{H}}\right.$ $\left./ \mathrm{cm}^{-2}\right)=23.9_{-0.2}^{+0.2}$, photon index $\Gamma=1.4_{\mathrm{NC}}^{+0.3}$, scattered fraction $f_{S}=11.7_{-6.4}^{+22.1} \%$, and $\mathrm{Fe} \mathrm{K} \alpha$ equivalent width upper limit of $\sim 0.6 \mathrm{keV}$. We find an unabsorbed $2-10 \mathrm{keV}$ luminosity of $4.6_{-0.6}^{+0.6} \times 10^{42} \mathrm{erg} \mathrm{s}^{-1}$.

5. Based upon our multiwavelength diagnostics, alternative scenarios-such as star formation activity, shock-driven emission, or photoionization by fewer than three AGNscannot effectively explain our observations.

Follow-up radio observations of SDSS J0849+1114 obtained with Very Long Baseline Interferometry (VLBI) are presented in Gabányi et al. (2019).

The presence of triple AGNs in hierarchical galaxy mergers is a natural consequence of $\Lambda \mathrm{CDM}$ predictions. Since late-stage mergers are predicted to be highly obscured (Blecha et al. 2018; Hopkins et al. 2008), the apparent dearth of dual and triple AGNs may in fact be due to the preselection strategy adopted to find them. With the serendipitous identification of one compelling case for a triple AGN originally selected using our WISE preselection strategy, the natural next step is to extend our study to identify further cases of triple AGNs using mid-infrared color selection. In a forthcoming investigation, we hope to examine a sample of WISE selected $(W 1[3.4 \mu \mathrm{m}]-W 2[4.6 \mu \mathrm{m}]>0.5)$ triple mergers in a systematic search for triple AGNs using an arsenal of optical, near-infrared, and X-ray diagnostics.

The authors thank the anonymous referee for a thoughtful review and constructive criticism of the manuscript. R.W.P. and S.S. gratefully acknowledge support from the Chandra Guest Investigator Program under NASA Grants GO6-17096X and GO7-18099X. R.W.P thanks the Chandra Help Desk for support during this project. R.W.P. thanks P. Boorman for the helpful discussions and assistance in troubleshooting the NUSTAR pipeline while R.W.P. became acquainted with the software. C.M.-K., R.O.S., and G.C. acknowledge support from the National Science Foundation, under grant number AST 1817233. C.R. acknowledges support from the CONICYT+PAI Convocatoria Nacional subvencion a instalacion en la academia convocatoria año 2017 PAI77170080. L.B. acknowledges support from the National Science Foundation under grant number AST-1715413.

This research made use of APLpy, an open-source plotting package for Python (Robitaille \& Bressert 2012), as well as the Ned Wright Cosmology Calculator (Wright 2006). We also gratefully acknowledge the use of the software TOPCAT (Taylor 2005) and Astropy (The Astropy Collaboration et al. 2018).

The LBT is an international collaboration among institutions in the United States, Italy, and Germany. LBT Corporation partners are The University of Arizona on behalf of the Arizona Board of Regents; Istituto Nazionale di Astrofisica, Italy; LBT Beteiligungsgesellschaft, Germany, representing the Max-Planck Society, The Leibniz Institute for Astrophysics Potsdam, and Heidelberg University; The Ohio State University, and The Research Corporation, on behalf of The University of Notre Dame, University of Minnesota and University of Virginia.

This publication makes use of data products from the Widefield Infrared Survey Explorer, which is a joint project of the University of California, Los Angeles, and the Jet Propulsion Laboratory/California Institute of Technology, funded by the National Aeronautics and Space Administration. Funding for SDSS-III has been provided by the Alfred P. Sloan Foundation, the Participating Institutions, the National Science Foundation, and the U.S. Department of Energy Office of Science. The SDSS-III website is http://www.sdss3.org/.

SDSS-III is managed by the Astrophysical Research Consortium for the Participating Institutions of the SDSS-III Collaboration including the University of Arizona, the Brazilian Participation Group, Brookhaven National Laboratory, Carnegie Mellon University, University of Florida, the French Participation Group, the German Participation Group, Harvard University, the Instituto de Astrofisica de Canarias, the Michigan State/Notre Dame/JINA Participation Group, Johns Hopkins University, Lawrence Berkeley National Laboratory, Max Planck Institute for Astrophysics, Max Planck Institute for Extraterrestrial Physics, New Mexico State University, New York University, Ohio State University, Pennsylvania State University, University of Portsmouth, Princeton University, the Spanish Participation Group, University of Tokyo, University of Utah, Vanderbilt University, University of Virginia, University of Washington, and Yale University. This research has made use of the NASA/IPAC Extragalactic Database (NED) which is operated by the Jet Propulsion Laboratory, California Institute of Technology, under contract with the National Aeronautics and Space Administration.

\section{Appendix}

In this section we show the full dynamic range of the optical spectra for the three galaxy nuclei within SDSS J0849+1114. 

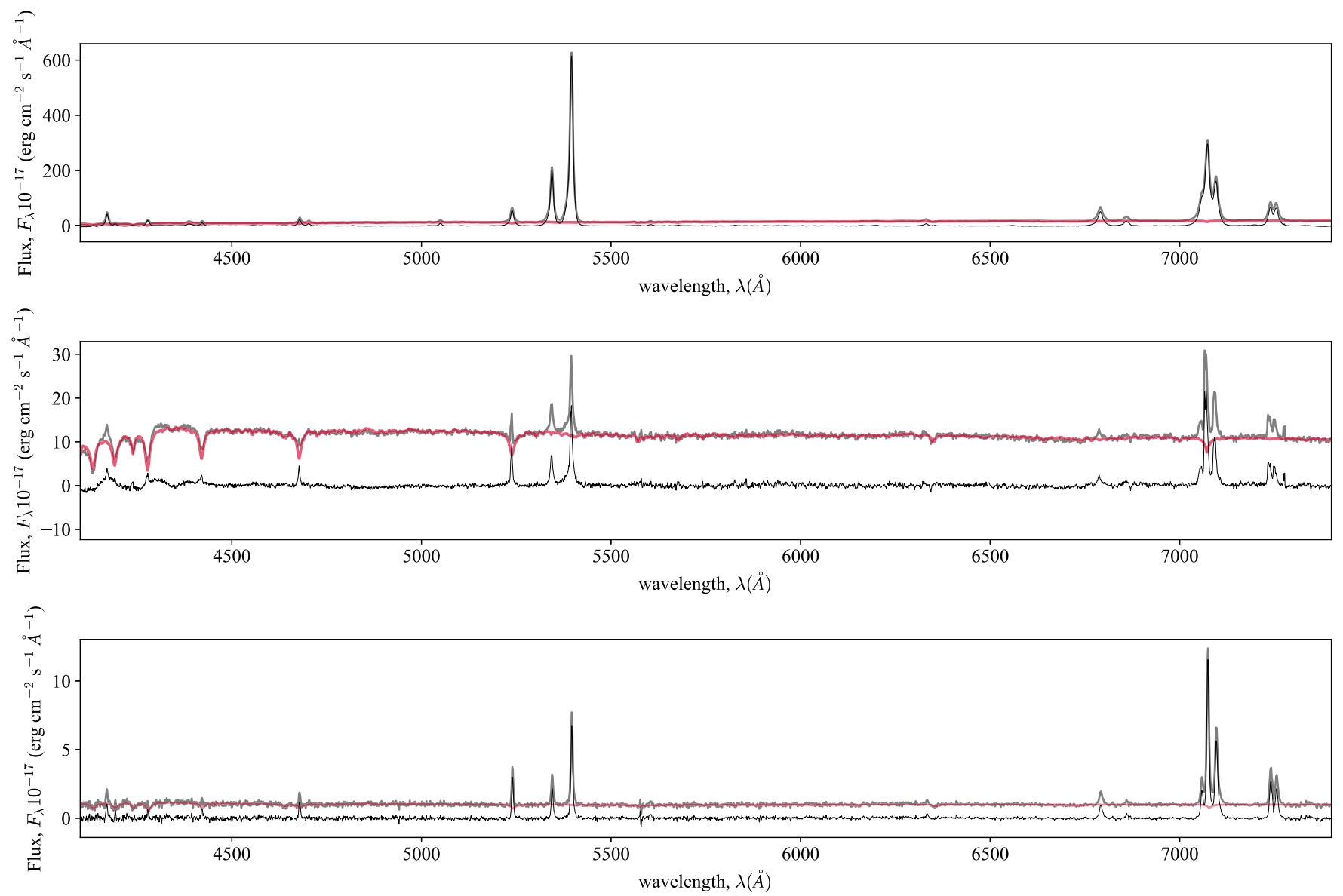

Figure 8. The full dynamic range of the optical spectra shown in Figure 2. Here, the top, middle, and bottom panels correspond to the spectra for Galaxies 1, 2, and 3, respectively. The spectra for Galaxies 1 and 2 were obtained with the LBT while the spectrum for Galaxy 3 was obtained by the SDSS. The spectra were fit using the procedure outlined in Section 2.6.1; the light gray spectrum in each panel shows the observed spectrum, the red line illustrates the spectrum of the best-fitting stellar population, and the black spectrum of each panel shows the resulting spectrum after subtracting the stellar population model.

\section{ORCID iDs}

Ryan W. Pfeifle (iD https://orcid.org/0000-0001-8640-8522 Shobita Satyapal (i) https://orcid.org/0000-0003-2277-2354 Christina Manzano-King (i) https://orcid.org/0000-00025253-9433

Jenna Cann (10) https://orcid.org/0000-0003-1051-6564 Remington O. Sexton (10 https://orcid.org/0000-00033432-2094

Barry Rothberg (iD https://orcid.org/0000-0003-2283-2185 Claudio Ricci (D) https://orcid.org/0000-0001-5231-2645 Laura Blecha (i) https://orcid.org/0000-0002-2183-1087 Sara L. Ellison (i) https://orcid.org/0000-0002-1768-1899 Mario Gliozzi (i) https://orcid.org/0000-0002-8818-9009 Nathan J. Secrest (iD https://orcid.org/0000-0002-4902-8077 Anca Constantin (ii) https://orcid.org/0000-0002-2441-1619

\section{References}

Allen, M. G., Groves, B. A., Dopita, M. A., Sutherland, R. S., \& Kewley, L. J. 2008, ApJS, 178, 20

Amaro-Seoane, P., Audley, H., Babak, S., et al. 2017, arXiv:1702.00786 Arnaud, K. A. 1996, in ASP Conf. Series 101, Astronomical Data Analysis Software and Systems V, 101, ed. G. H. Jacoby \& J. Barnes, 17

Baloković, M., Brightman, M., Harrison, F. A., et al. 2018, ApJ, 854, 42 Barnes, J. E., \& Hernquist, L. 1992, ARA\&A, 30, 705

Barth, A. J., Bentz, M. C., Greene, J. E., \& Ho, L. C. 2008, ApJL, 683, L119
Bennert, N., Jungwiert, B., Komossa, S., Haas, M., \& Chini, R. 2006, NewAR, 50,708

Bianchi, S., Piconcelli, E., Pérez-Torres, M. Á., et al. 2013, MNRAS, 435, 2335

Blecha, L., Snyder, G. F., Satyapal, S., \& Ellison, S. L. 2018, MNRAS, 478, 3056

Bonetti, M., Perego, A., Capelo, P. R., Dotti, M., \& Miller, M. C. 2018, PASA, 35, e017

Bonetti, M., Sesana, A., Haardt, F., Barausse, E., \& Colpi, M. 2019, MNRAS, 486, 4044

Brightman, M., \& Nandra, K. 2011, MNRAS, 413, 1206

Cappellari, M. 2017, MNRAS, 466, 798

Cash, W. 1979, ApJ, 228, 939

Colina, L., Arribas, S., \& Monreal-Ibero, A. 2005, ApJ, 621, 725

Comerford, J. M., Pooley, D., Barrows, R. S., et al. 2015, ApJ, 806, 219

Deane, R. P., Paragi, Z., Jarvis, M. J., et al. 2014, Natur, 511, 57

Djorgovski, S. G., Courbin, F., Meylan, G., et al. 2007, ApJL, 662, L1

Donley, J. L., Kartaltepe, J., Kocevski, D., et al. 2018, ApJ, 853, 63

Dopita, M. A., \& Sutherland, R. S. 1995, ApJ, 455, 468

Ellison, S. L., Secrest, N. J., Mendel, J. T., Satyapal, S., \& Simard, L. 2017, MNRAS, 470, L49

Ellison, S. L., Viswanathan, A., Patton, D. R., et al. 2019, MNRAS, 487, 2491

Farina, E. P., Montuori, C., Decarli, R., \& Fumagalli, M. 2013, MNRAS, 431, 1019

Foreman-Mackey, D., Hogg, D. W., Lang, D., \& Goodman, J. 2013, PASP, 125,306

Gabányi, K. E., Frey, S., Satyapal, S., Constantin, A., \& Pfeifle, R. W. 2019, A\&A, 630, L5

Goulding, A. D., Greene, J. E., Bezanson, R., et al. 2018, PASJ, 70, S37

Hainline, K., Hickox, R. C., Greene, J. E., et al. 2014, ApJ, 787, 65

Hainline, K. N., Hickox, R., Greene, J. E., Myers, A. D., \& Zakamska, N. L. 2013, ApJ, 774, 145 
Heckman, T. M., Miley, G. K., van Breugel, W. J. M., \& Butcher, H. R. 1981, ApJ, 247, 403

Hibbard, J. E., \& van Gorkom, J. H. 1996, AJ, 111, 655

Ho, L. C., Filippenko, A. V., \& Sargent, W. L. W. 1997, ApJS, 112, 315

Hoffman, L., \& Loeb, A. 2007, MNRAS, 377, 957

Hopkins, P. F., Cox, T. J., Kereš, D., \& Hernquist, L. 2008, ApJS, 175, 390

Kalfountzou, E., Santos Lleo, M., \& Trichas, M. 2017, ApJL, 851, L15

Kauffmann, G., Heckman, T. M., Tremonti, C., et al. 2003, MNRAS, 346, 1055

Kelley, L. Z., Blecha, L., Hernquist, L., Sesana, A., \& Taylor, S. R. 2017, MNRAS, 471, 4508

Kewley, L. J., Dopita, M. A., Sutherland, R. S., Heisler, C. A., \& Trevena, J. 2001, ApJ, 556, 121

Kewley, L. J., Groves, B., Kauffmann, G., \& Heckman, T. 2006, MNRAS, 372, 961

Kocevski, D. D., Brightman, M., Nandra, K., et al. 2015, ApJ, 814, 104

Koss, M., Mushotzky, R., Treister, E., et al. 2012, ApJL, 746, L22

Koss, M. J., Blecha, L., Bernhard, P., et al. 2018, Natur, 563, 214

Lansbury, G. B., Alexander, D. M., Del Moro, A., et al. 2014, ApJ, 785, 17

Lanzuisi, G., Civano, F., Marchesi, S., et al. 2018, MNRAS, 480, 2578

Leitherer, C., Ekström, S., Meynet, G., et al. 2014, ApJS, 212, 14

Leitherer, C., Schaerer, D., Goldader, J. D., et al. 1999, ApJS, 123, 3

Liu, X., Shen, Y., Strauss, M. A., \& Hao, L. 2011, ApJ, 737, 101

Manzano-King, C., Canalizo, G., \& Sales, L. 2019, arXiv:1905.09287

Marchesi, S., Ajello, M., Marcotulli, L., et al. 2018, ApJ, 854, 49

Mihos, J. C., \& Hernquist, L. 1996, ApJ, 464, 641

Mingarelli, C. M. F., Lazio, T. J. W., Sesana, A., et al. 2017, NatAs, 1, 886

Nelson, C. H., \& Whittle, M. 1996, ApJ, 465, 96

Pfeifle, R. W., Satyapal, S., Secrest, N. J., et al. 2019, ApJ, 875, 117

Ricci, C., Bauer, F. E., Treister, E., et al. 2016, ApJ, 819, 4

Ricci, C., Bauer, F. E., Treister, E., et al. 2017, MNRAS, 468, 1273

Rich, J. A., Kewley, L. J., \& Dopita, M. A. 2011, ApJ, 734, 87

Robitaille, T., \& Bressert, E. 2012, APLpy: Astronomical Plotting Library in Python, Astrophysics Source Code Library, ascl:1208.017

Rodríguez Zaurín, J., Tadhunter, C. N., Rose, M., \& Holt, J. 2013, MNRAS, 432,138
Rothberg, B., \& Joseph, R. D. 2004, AJ, 128, 2098

Ryu, T., Perna, R., Haiman, Z., Ostriker, J. P., \& Stone, N. C. 2018, MNRAS, 473,3410

Satyapal, S., Ellison, S. L., McAlpine, W., et al. 2014, MNRAS, 441, 1297

Satyapal, S., Secrest, N. J., Ricci, C., et al. 2017, ApJ, 848, 126

Schawinski, K., Urry, M., Treister, E., et al. 2011, ApJL, 743, L37

Schweizer, F. 1982, ApJ, 252, 455

Schweizer, F. 1996, AJ, 111, 109

Secrest, N. J., Satyapal, S., Gliozzi, M., et al. 2015, ApJ, 798, 38

Sesana, A., Haardt, F., Madau, P., \& Volonteri, M. 2004, ApJ, 611, 623

Sexton, R. O., Canalizo, G., Hiner, K. D., et al. 2019, ApJ, 878, 101

Stern, D., Assef, R. J., Benford, D. J., et al. 2012, ApJ, 753, 30

Stickley, N. R., \& Canalizo, G. 2014, ApJ, 786, 12

Sutton, A. D., Roberts, T. P., Walton, D. J., Gladstone, J. C., \& Scott, A. E. 2012, MNRAS, 423, 1154

Taylor, M. B. 2005, in ASP Conf. Ser. 347, Astronomical Data Analysis Software and Systems XIV, ed. P. Shopbell, M. Britton, \& R. Ebert (San Francisco, CA: ASP), 29

The Astropy Collaboration, Price-Whelan, A. M., Sipőcz, B. M., et al. 2018, AJ, 156, 123

Toomre, A., \& Toomre, J. 1972, ApJ, 178, 623

Vacca, W. D., Cushing, M. C., \& Rayner, J. T. 2003, PASP, 115, 389

Valdes, F., Gupta, R., Rose, J. A., Singh, H. P., \& Bell, D. J. 2004, ApJS, 152,251

Veilleux, S., Cecil, G., Bland-Hawthorn, J., \& Shopbell, P. L. 2002, RMxAC, 13,222

Verbiest, J. P. W., Lentati, L., Hobbs, G., et al. 2016, MNRAS, 458, 1267

Véron-Cetty, M. P., Joly, M., \& Véron, P. 2004, A\&A, 417, 515

von der Linden, A., Wild, V., Kauffmann, G., White, S. D. M., \& Weinmann, S. 2010, MNRAS, 404, 1231

Weston, M. E., McIntosh, D. H., Brodwin, M., et al. 2017, MNRAS, 464, 3882

Woo, J.-H., Bae, H.-J., Son, D., \& Karouzos, M. 2016, ApJ, 817, 108

Wright, E. L. 2006, PASP, 118, 1711

Wrobel, J. M., Walker, R. C., \& Fu, H. 2014, ApJL, 792, L8

Wyithe, J. S. B., \& Loeb, A. 2003, ApJ, 590, 691

Zamanov, R., Marziani, P., Sulentic, J. W., et al. 2002, ApJL, 576, L9 